\title{
Concepts of Operations for Asteroid Rendezvous Missions Focused on Resources Utilization
}

\author{
Robert P. Mueller ${ }^{1}$, Laurent Sibille ${ }^{2}$, Gerald B. Sanders ${ }^{3}$, Christopher A. Jones ${ }^{4}$ \\ ${ }^{1}$ NASA, Surface Systems Office, NE-S, Kennedy Space Center, KSC, FL 32899 \\ ${ }^{2}$ Enterprise Advisory Services Inc. (ESC), Applied Sciences and Technology, Kennedy Space \\ Center, FL 32899 \\ ${ }^{3}$ NASA, Propulsion and Power Division, EP3, Johnson Space Center, Houston, TX 77058 \\ ${ }^{4}$ NASA, Space Mission Analysis Branch, Langley Research Center, Hampton, VA 23681
}

\begin{abstract}
Several asteroids are the targets of international robotic space missions currently manifested or in the planning stage. This global interest reflects a need to study these celestial bodies for the scientific information they provide about our solar system, and to better understand how to mitigate the collision threats some of them pose to Earth. Another important objective of these missions is providing assessments of the potential resources that asteroids could provide to future space architectures.

In this paper, we examine a series of possible mission operations focused on advancing both our knowledge of the types of asteroids suited for different forms of resource extraction, and the capabilities required to extract those resources for mission enhancing and enabling uses such as radiation protection, propulsion, life support, shelter and manufacturing. An evolutionary development and demonstration approach is recommended within the framework of a larger campaign that prepares for the first landings of humans on Mars. As is the case for terrestrial mining, the development and demonstration approach progresses from resource prospecting (understanding the resource, and mapping the 'ore body'), mining/extraction feasibility and product assessment, pilot operations, to full in-situ resource utilization (ISRU). Opportunities to gather specific knowledge for ISRU via resource prospecting during science missions to asteroids are also examined to maximize the pace of development of needed ISRU capabilities and technologies for deep space missions.
\end{abstract}

\section{INTRODUCTION}

In April 2010, President Obama announced a new challenge for NASA: send an astronaut crew to an asteroid by 2025 to spur the development of deep space capabilities for human missions and to better understand the nature of potentially hazardous asteroids. NASA has developed mission concepts that would capture either an entire asteroid or take a boulder from an asteroid and bring the object to a stable lunar orbit where astronauts will travel to study it. This robotic mission that will encounter the asteroid in deep space and redirect it to the vicinity of the Moon is known as the Asteroid Redirect Robotic Mission (ARRM). The human missions that will follow are part of a strategic campaign that will carry humans to Mars on a sustainable pioneering enterprise to establish our presence in space. This Evolvable Mars Campaign (EMC) aims to develop the operational foundation and critical technologies through a series of evolutionary deep space missions that will enable long duration crewed stays on the Mars surface and in the Martian system. Orbital relationships between Earth and Mars constrain the durations 
of travel between the two planets and point to a long surface stay ca. 500 days on Mars as the more favorable option in terms of energy and crew protection from deep space radiation. The first humans to explore Mars will then need to embrace the pioneering spirit of using advanced technologies to make use of resources around them and to create solutions for a safe sustained presence.

The visionary approach of the EMC provides a compelling context to use the asteroid made accessible by ARRM in lunar distant retrograde orbit (LDRO) to develop and demonstrate ISRU technologies and concepts of operations in space. This paper describes how the detection, prospecting, extraction and utilization of asteroid resources is essential to understanding the threat they pose our planet, how to mitigate that threat, and how to fulfill the pioneering ambitions of mankind's future in deep space by utilizing their resources.

\section{EXPLORING ASTEROID RESOURCES: A long-term Investment for Mankind's Future}

In Situ Resource Utilization represents a change in the paradigm of space exploration. The vast volatile and mineral resources that make up every object in the Solar System can become the source of new life support consumables for humans (water, oxygen, nitrogen) or propellants (hydrogen, methane, oxygen, carbon dioxide), and even material feedstock for additive 3D manufacturing and construction.

Asteroids are a very diverse group of celestial objects exhibiting wide ranges of size, origin of formation, and orbital path. While the large majority of asteroids orbit the Sun between the orbits of Mars and Jupiter (the asteroid belt), some are dislodged through collisions and planetary influences and their new orbits may cross the orbit of the Earth (Apollo, Amor, and Aten asteroids). If their paths bring them within 0.3 astronomical units (AU), they are listed among near-Earth objects (NEO). An estimated $20 \%$ of NEOs represent a potential threat to Earth because they come within 0.05 AU of Earth's orbit (NRC, 2010). The large numbers of asteroids in the Solar System have long been considered a source of a variety of resources for humanity when it will venture out and begin settling beyond Earth. Their varied physical, chemical, and mineral compositions have been studied extensively for years through the systematic examination of thousands of collected meteorites that have fallen on Earth (Britt, 2014). However, only asteroids that can survive entry and impact are available for study; this may bias and limit our understanding of asteroid composition. More recently, sophisticated space missions have successfully reached several asteroids and maintained precise formation flying near them. This has enabled long scientific observations and even sampling of selected asteroids. Table 1 presents a succinct summary of current knowledge of the mineral characteristics of asteroid types that could offer valuable resources (Britt, 2014; SBAG, 2014).

Table 1. Expected resources of value in asteroid types

\begin{tabular}{|l|l|l|}
\hline Asteroid Type & Description (major surface minerals) & \multicolumn{2}{|l|}{ Expected resources } \\
\hline $\mathrm{C}$ (carbonaceous) & Clays, organics & $\begin{array}{l}\text { Water (hydrated minerals) }-10-20 \mathrm{wt} \% \\
\text { Simple hydrocarbons, metals }\end{array}$ \\
\hline $\mathrm{D}, \mathrm{P}$ & Organics, Anhydrous silicates (?) & Volatiles, water \\
\hline $\mathrm{M}$ (metal) & Metal, enstatite & Fe, Ni, Pt-group metals \\
\hline $\mathrm{S}$ (stony) & Olivine, pyroxene, metal & Oxides, oxygen, metals \\
\hline
\end{tabular}


An unprecedented convergence of interests exists today worldwide about asteroids and how they may affect our future. NASA was founded in part with a congressional mandate (NASA Auth. Act, 1958) stating that "the unique competence of the Administration be directed to detecting, tracking, cataloguing, and characterizing near-Earth asteroids and comets in order to provide warning and mitigation of the potential hazard of such near-Earth objects to the Earth." The program of detection and warning has been renewed in intensity and funding in the past 10 years with congressional mandates (NASA Auth. Act, 2005) regarding the completion of asteroid inventories for certain class sizes at specific dates (e.g., 90\% of objects larger than $140 \mathrm{~m}$ by 2020). In 2010, the U.S. presidential declaration to send astronauts to study an asteroid was later accompanied by the creation of a grand challenge by NASA to understand and mitigate the threat that asteroids pose. In 2012, the European Union initiated the NEOShield project and began funding an international study team to define three techniques (kinetic impactor, gravity-tractor, and blast deflection) to deflect large asteroids from their collision path with Earth (Harris, 2013).

The renewed concerns of U.S. elected officials about asteroids coincide with international advances in detection and image analysis technologies that have identified potentially hazardous objects (PHO) that will nearly miss Earth within the next few decades. In 2013, the explosive entry of a near-Earth asteroid of 15-20 $\mathrm{m}$ in diameter (stony LL chondrite) into Earth's atmosphere over the Russian city of Chelyabinsk made the global population more aware that such an event can indeed occur and cause direct and significant damages to people and cities.

In the U.S., work is ongoing on the ARRM mission to reach an asteroid, capture it or a large boulder from it (Robotic Boulder Capture option), and redirect it to place it in a stable LDRO orbit. A human mission with the Orion spacecraft to study the captured asteroid in its new location would then follow the robotic mission. These two missions are among several international missions pursuing in-situ scientific studies of asteroids and comets of different types and acquiring samples (Hayabusa, OSIRIS-REx, Hayabusa II, Dawn, Rosetta). Advanced reliable deep space robotics and propulsion have enabled scientists around the world to be in a position to examine a variety of asteroid types in their original state and deepen their understanding far beyond what they have learned so far from fallen and retrieved meteorites.

The feasibility of carrying out such missions for scientific purposes has also spurred interest by U.S. commercial companies. Planetary Resources Inc. and Deep Space Industries were created recently to target and ultimately exploit asteroids with valuable resources in space for both inspace use and terrestrial markets.

NASA also sees new knowledge of asteroids and the resources they offer as a part of a larger vision of human and robotic activities in space in the $21^{\text {st }}$ century. The information gathered by science missions to the asteroids will inform the human missions to the asteroid captured in LDRO that are elements of the larger EMC. The campaign is a set of mission architectures that develop technologies and deep space capabilities culminating in the sustained presence of humans on Mars with the ability to rely on local resources for their safe stay and return to Earth (Fig. 1). 


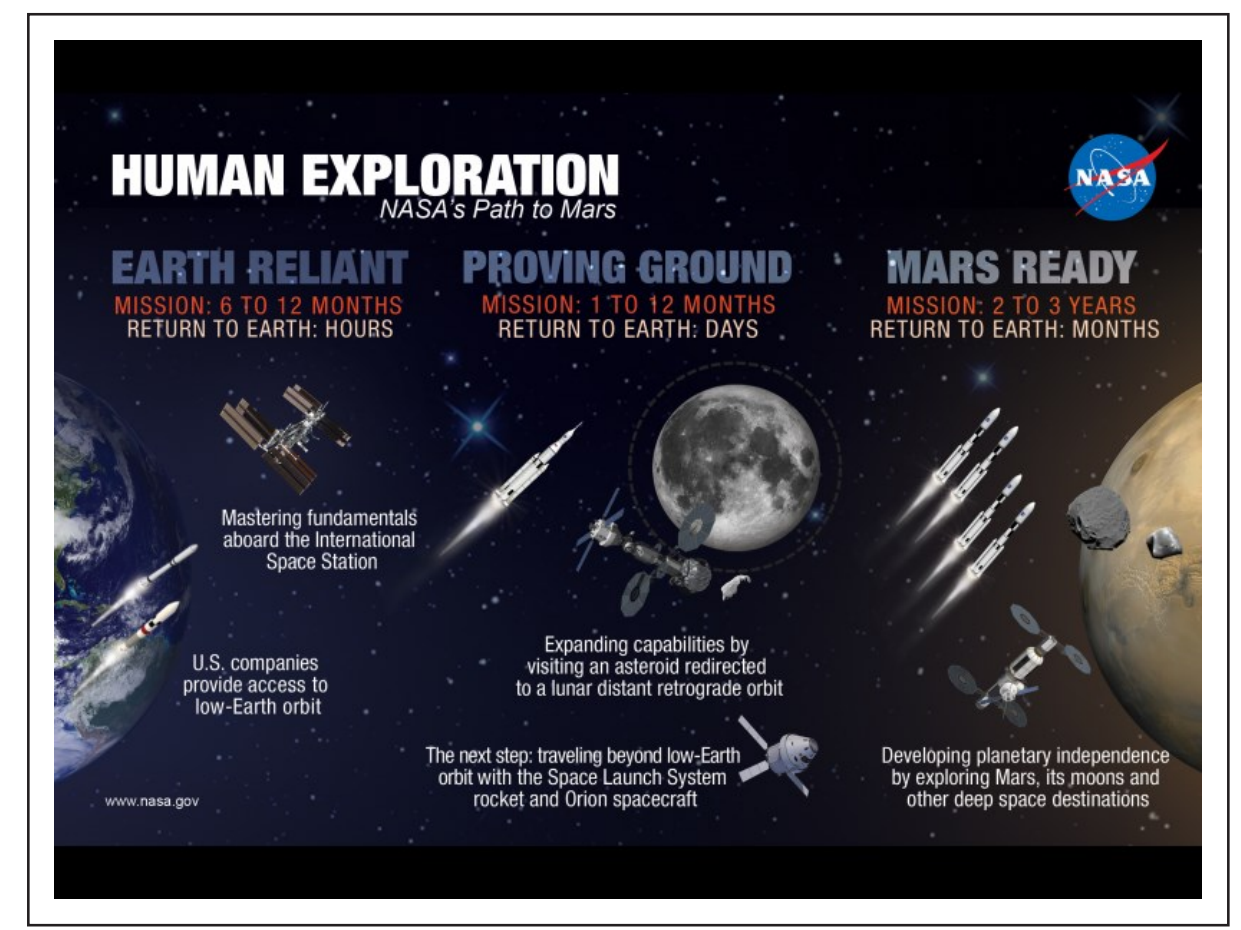

Figure 1. NASA's Path to a Mars Exploration Campaign.

Mars is a deep space destination beyond the Moon where humans can aspire both to survive and to settle. The relative positions of Earth and Mars and associated orbital mechanics make the journey to Mars and back orders of magnitude longer than the ones made to the Moon; thus, the technical requirements and constraints, as well as the risks, are orders of magnitude greater. The achievement of a sustained and safe presence of humans on a planet months away of travel from Earth is more likely to succeed if the crew is equipped with the technical ability and know-how to become self-reliant. In pioneering situations on Earth, the knowledge and tools to access local resources and transform them into needed consumables or items has made the difference between a thriving endeavor and its demise. Since other planets are not readily habitable and humans are not physiologically adapted to space environments, this need for self-reliance becomes even more important. Asteroids offer many resources that can meet those needs in space and that are not locked in deep gravity wells. The insertion of asteroid missions in the path to Mars create a unique synergy between the needs to understand these objects, to mitigate the threat some pose to Earth, and to gain the ability to become self-reliant in the unforgiving environment of deep space.

\section{MISSIONS OF OPPORTUNITY: Advancing ISRU by Serving High Priority Missions}

ISRU technologies have been developed for many years and achieved successful demonstrations in laboratories and field deployments at analog sites (Larson, 2011). Despite those numerous successes, it has been difficult to find space missions that could accept the risk of deploying such new paradigm-changing technologies to meet their objectives. The insertion of ISRU in the critical path of a space mission contains significant risks even when ISRU can multiply its capability (e.g., extending mission life via the production of in-situ propellant). 
The stated urgency to understand asteroids and their nature and the goal of pioneering human stays on Mars places a new spotlight on the approach of discovering and using space resources to meet those needs. Moreover, the suite of planned missions to asteroids and the EMC architectures under study offer the opportunity to use, validate, and qualify ISRU technologies in flight by serving the missions' objectives as part of an evolution toward reliance on ISRU instead of taking high risks. The following mission concepts are built on that idea to help missions succeed and enable the confident adoption of ISRU in space activities in the long-term.

NASA's Origins-Spectral Interpretation-Resource Identification-Security-Regolith Explorer (OSIRIS-REx) mission will follow in the footsteps of NASA's Stardust mission and JAXA's Hayabusa mission: it will return a sample of another body to Earth (Drake, 2011). The target for OSIRIS-REx is Bennu (1999 RQ36), a carbonaceous asteroid. The spacecraft, scheduled for launch in 2016, is expected to arrive at Bennu in 2019 to map its surface; it will then acquire a sample to be returned to Earth in 2023. In its current mission plan, OSIRIS-REx will greatly enhance our knowledge of carbonaceous asteroid material and the resources they contain via comprehensive surface multi-spectral observations and radio science. Its emphasis on the search and characterization of surface volatile plumes and their mineral and chemical context in the surface material will be of great value for future ISRU use. Additional high-value knowledge of such volatile resources would be gained if the science team were to select a potential volatile plume site as the source of the sample to be returned to Earth. The sampling activity also could offer a valuable opportunity for ISRU; the touch and go sample acquisition mechanism (TAGSAM) is designed to acquire regolith material using $\mathrm{N}_{2}$ gas to lift granular material. In so doing, it is expected to create a small crater that would reveal the local subsurface structure of the asteroid (Metzger, 2014), and the use of the onboard high definition cameras to document the spectral information of the freshly excavated area would be of tremendous value for ISRU.

JAXA intends to follow up their successful Hayabusa mission with Hayabusa 2, another sample return mission to an asteroid (Tsuda, 2013). The target is $1999 \mathrm{JU} 3$, a carbon rich C-type asteroid that may provide information on the early solar system. The mission is targeted for a launch in late 2014, with an estimated arrival date in 2018 and a sample return date in 2020. The use of kinetic impactors to lift regolith and capture it during ballistic flight will be repeated on this mission with a carbonaceous chondrite body after seeing some success with Hayabusa on the stony Itokawa rubble pile asteroid. A cratering experiment will be observed by a free-flying camera (DCAM3) and the spacecraft will later observe the expected 2-m diameter crater from its home position, $20 \mathrm{~km}$ above the surface. Similarly to the OSIRIS-REx mission, it is hoped that the spacecraft can spectrally image the crater at low altitude for higher resolution imagery to reveal the subsurface structure and better inform the creation of asteroid-scale resource maps. The deployment of the small (10 kg) hopping landing package Mobile Asteroid Surface Scout (MASCOT) for detailed up-close surface science observations at 3 sites and 3 micro rovers (MINERVA A1, A2 and B) weighing $\sim 1 \mathrm{~kg}$ each will test low-gravity surface mobility and will gather a wealth of information for ISRU engineering. 
The ARRM mission (Brophy, 2013; Muirhead, 2014; Mazanek, 2014) is currently under consideration by NASA to study and return some or all of an asteroid to cis-lunar space before 2025 (Fig. 2).

The selection of the target asteroid has yet to be made but a short list of candidates has emerged. The Small Bodies Assessment Group (SBAG) has recently completed their report on the respective value of selected asteroid types for the ARRM mission objectives (Chabot, 2014). The stated objectives of the ARRM are not scientific in nature but aim more at realizing the engineering capability of seizing large asteroid material and moving it across space. Since astronaut crews in LDRO will then explore the asteroid as part of the evolution of deep space missions toward Mars landings, the asteroid must also be of scientific value. In addition, the arrival and stable residence of the captured asteroid near the Moon represents a unique opportunity to deploy ISRU pathfinder technologies on an asteroid in a series of missions that can carry enough hardware mass to execute end-to-end resource processing within the capabilities of the NASA Space Launch System (SLS) and EMC architecture under development. The authors of the report (Chabot, 2014) acknowledged such views and strongly recommended the selection of a volatile-rich carbonaceous C-type asteroid, to provide the opportunity to perform the widest range of ISRU activities possible at the asteroid. They also note that the development of autonomous resource extraction experiments are not expected to be complete by

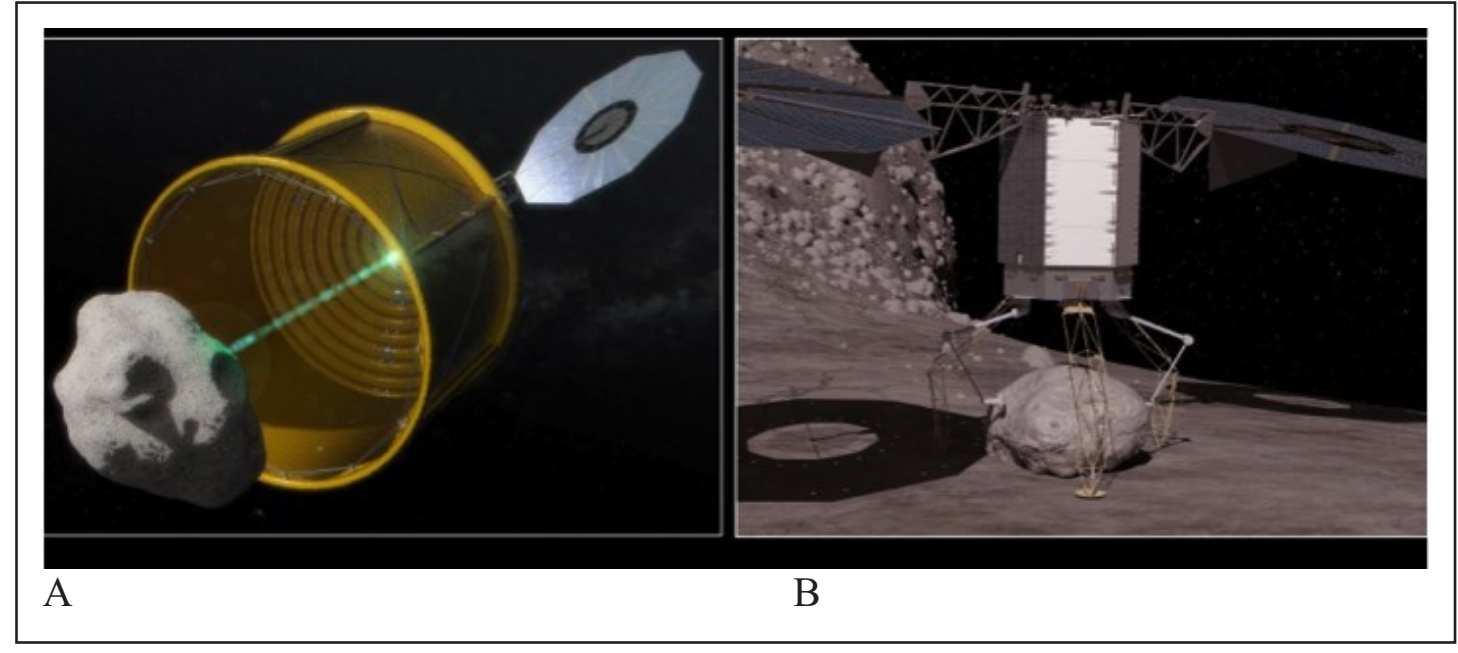

Figure 2. NASA's leading ARRM concepts for whole asteroid capture (A) and Robotic Boulder Capture (B). (Courtesy NASA for (A) and NASA / AMA Inc. for (B))

the time the ARRM is launched unless immediate investment is initiated. However, the ARRM provides an opportunity to perform the first step of future ISRU activities during the transit journey to LDRO: the deployment of detection equipment capable of identifying the internal resources of the asteroid on a large scale. The time afforded by the transit journey would allow a long period of data collection by radar or other ground penetrating techniques that would serve both the crew exploration of the asteroid and ISRU operations after arrival at LDRO.

\section{EVOLUTIONARY ISRU TECHNOLOGY DEVELOPMENT}

The gradual insertion of ISRU technologies and concepts of operations into the critical path of deep space missions should follow a systematic progression of capabilities demonstrated through 
increasingly challenging environments. NASA's EMC enables this type of technological evolution for the first time by using ground test facilities, current in-space assets such as the International Space Station (ISS), planned asteroid and lunar science missions, and pathfinder missions in LDRO and at Mars in the coming decades. Early ISRU prospecting and processing tests on a carbonaceous C-type asteroid at LDRO could lay the groundwork for harvesting resources from the Mars moon Phobos that appears to have similar characteristics.

Prospecting for resources. Understanding what resources exist, their form, concentration, and distribution is essential to harvesting and utilizing these resources in future missions. Development of the technologies needed for asteroid resource prospecting will leverage both the Curiosity rover Sample Analysis on Mars (SAM) instrument (Leshin, 2013) and the Regolith and Environment Science \& Oxygen and Lunar Volatile Extraction (RESOLVE) instrument under development for the NASA Resource Prospector (RP) mission (Sanders, 2012). In both instruments, small soil/regolith samples are extracted using a drill and heated in an oven; the released volatile gases are analyzed with a gas chromatograph/mass spectrometer. Modifying these processes for operation in microgravity will require work described in the following subsections.

Ground Testing and Microgravity Testing. Extensive ground testing of sub-scale to near-full scale ISRU hardware has been taking place at NASA facilities, universities, companies, and international institutions. The number of facilities that can create relevant environments (beds of regolith and high-fidelity resource simulants such as ice and volatiles under vacuum, thermal environment) is very limited and require more investments to accommodate the needs of prototype hardware and their functions. Terrestrial analog testing provides valuable data on issues such as repeated handling of regolith, end-to-end coordinated processes, and teleoperation practices (Larson, 2011). This ground development process must continue unabated and technologies must transition as soon as possible to a microgravity environment where complex issues that cannot be simulated well on the ground should be tackled. The ISS is currently projected to continue operations possibly until 2024 and provides an ideal platform for extended testing of ISRU technology in preparation for space mission use. The absence of significant gravity during operations at an asteroid changes not only the nature and behavior of the regolith and the bound resources but also the excavation, handling, and transporting of such material during which reaction forces and momentum conservation are dominant. Similarly, the processing of regolith and resources in reactors where gases, liquids, and solids mix and interact is affected in multiple ways by the microgravity environment.

Exploration Augmentation Module. While the ISS currently offers a variety of experiment racks and supporting capabilities to perform self-contained ISRU processes on a small-scale, the addition of an external carrier platform capability would allow an extensive technology maturation program for ISRU technologies on all processes and at the proper scale. An Exploration Augmentation Module (EAM) is under consideration and the EMC could accommodate ISRU activities as well as other systems with similar needs for technology maturation. Some ISRU processes, such as the conversion of trash to propellant reagents or consumables, can be performed inside a habitat with crew present. In addition, the 'dirty' and hazardous nature of space resource processing activities and the scale of end-to-end processes (from regolith acquisition to delivery and storage of the targeted resources) will often require that they be conducted outside crew modules and exposed to the space environment. ISRU hardware designed to access, acquire, transport, and process asteroid regolith would undergo testing and 
validation on an external rack or platform where the combination of microgravity, vacuum, and thermal constraints affects each process and the processed materials. The "open-to-space" capability would also be able to accommodate larger subsystems undergoing scaling tests.

Asteroid Retrieval Crew Mission (ARCM) and asteroid ISRU in cis-lunar space. In the next phase, ISRU technologies would follow their evolutionary path toward deep space implementation when an EAM could be brought to the lunar orbit where the captured asteroid will be residing at the completion of the ARRM mission. As previously described, significant data on the resources of the asteroid could have been gathered and analyzed during the ARRM mission to inform what type of ISRU technologies are best suited to work with the available asteroid. A variety of concepts of operations are under consideration for ISRU activities with the redirected asteroid.

- ISRU assets installed externally to the EAM can be tele-operated by the astronauts during one of the ARCM missions, including surface rovers, landers or surface equipment emplaced by robotic arms for prospecting and sampling. The material can then be brought to reactors on the EAM external ISRU processing platform for resource extraction, analysis, storage and conditioning.

- In more matured versions, autonomous equipment can perform some activities such as continued prospecting, new operations practices, and long-duration systems testing in deep space while the crew is absent. Tele-operations from Earth are also possible during that stage to maximize the testing time and to advance knowledge of systems behaviors during long exposure in space.

- The selection of the asteroid for the ARRM mission will play a defining role in what type of ISRU can be demonstrated and validated for future implementation in Mars missions. The selection of a volatile-rich carbonaceous C-type would enable the prospecting and extraction of water ( 10-20 wt.\% estimated), and perhaps processing the asteroidal material into inspace fabricated radiation shielding panels that can protect deep space habitats and/or spacecraft on their way to Mars.

ISRU for Mars Transit and Phobos missions. The technology maturation program will culminate in the flight qualification of ISRU hardware specifically selected for use on missions to the Mars system. The deep space habitat that will carry a crew during its long transit to Mars destinations such as Phobos, Mars orbit or the Mars surface would benefit from advanced technologies converting on board trash into propellant (methane, oxygen) or consumables (breathing oxygen, water), and extracted asteroidal material into radiation shielding. The possible visit of a crew to Phobos could make a direct use of the propellant made during transit as a way to keep a station orbit near the Mars moon and the synergistic use of ISRU technologies on a C-type asteroid at LDRO could directly apply to resource extraction at Phobos on similar materials.

Mars Pathfinder missions and Mars Surface missions. The use of atmospheric $\mathrm{CO}_{2}$ capture and processing technologies onboard the Mars 2020 rover is an important step taken by NASA toward validating ISRU technologies with potential for large-scale implementation in Mars surface human missions. The concept of processing the Martian atmosphere (containing over 95\% carbon dioxide) to extract oxygen for the propulsion of a crew ascent vehicle for a return to Earth has been studied extensively and is under consideration for the EMC. The production and 
storage of oxygen would need to be done autonomously by ISRU surface assets ahead of the arrival of a crew. A similar concept would see the extraction of water $\left(\mathrm{H}_{2} \mathrm{O}\right.$ or $\left.\mathrm{OH}\right)$ from the regolith and its conversion into oxygen and hydrogen, the latter being then used in a Sabatier reaction with carbon dioxide for the production of methane as the fuel for the crew Mars ascent stage rocket engine.

The development path for ISRU technologies within the Mars campaign will culminate in these critical applications and others like the use of extracted water for life support or additional exploration capabilities, the on-site construction of landing areas by consolidation of regolith, the creation of habitat protection against radiation, and the protection of other arriving spacecraft from landing debris. as well as in-situ manufacturing from in-situ derived feedstocks.

Public-Private Partnerships and Commercial ISRU at Asteroids. Governments are not the only interested partners in the development of technologies for the utilization of space resources. The inclusion of asteroid missions in the EMC invites a strong synergy between private and government interests to combine efforts and maximize the use of data and techniques. The presence of a captured asteroid with valuable resources in a stable lunar orbit may prove attractive for companies aiming to mine such objects. The development of ISRU technologies in the proving ground years can be done with shared financial commitments and contractual arrangements that benefit every partner. The Mars-forward momentum will likely carry the public interests away from the captured asteroid that could in turn become a prime mining location for private entities. The legacy of the captured asteroid becoming the source of accessible space resources could in time be the ignition spark of an emerging, new space-based economy.

\section{CONCLUSION}

The current heightened awareness of asteroids by many entities and people on Earth, and the unique convergence of interests from international space agencies, governments and space entrepreneurs have created a favorable context for using space resources as an enabling technology for future space activities. NASA's Evolvable Mars Campaign under study offers a clear opportunity to develop these remarkable and "game-changing" technologies in a synergistic manner with other mission priorities including those of private technology developers. The progressive evolution of ISRU technologies and concepts of operations makes use of current assets at the ISS, augments the value of planned missions to asteroids including the ARRM, and supports the required qualification of Mars-forward ISRU critically needed for safe, sustainable pioneering on the red planet.

\section{REFERENCES}

Britt, D. T., Guy Consolmagno, S. J., and Lebofsky, L. (2014). "Chapter 26: Main-Belt Asteroids." Encyclopedia of the Solar System, Eds. Spohn,T., Breuer, D., and Johnson, T., Elsevier, 583-601.

Brophy, J. R., and Muirhead, B. (2013). "Near-Earth Asteroid Retrieval Mission (ARM) study." $33^{\text {rd }}$ International Electric Propulsion Conference, Washington, D.C., IEPC-2013-82.

Chabot, N., Abell, P., Britt, D., Dankanich, J., Emery, J., Rivkin, A., and Sheeres, D. (2014). "Report of the Small Bodies Assessment Group Asteroid Redirect Mission

Special Action Team." Lunar and Planetary Institute./ SBAG website, http://www.lpi.usra.edu/sbag/documents/SBAG ARM SAT 0730 14.pdf (7/30/14). 
Drake, M. J., Lauretta, D. S., Team, O. (2011). “OSIRIS-REx Asteroid Sample Return Mission.” American Geophysical Union, Fall Meeting 2011, abs. \#P42A-03.

Harris, A. W., et al. (2013). "The European Union funded NEOShield project: a global approach to near-Earth object impact threat mitigation." Acta Astronautica 90(1), 80-84.

Larson, W. E., Sanders, G. B., and Hyatt, M. (2011). "ISRU - From Concept to Reality: NASA Accomplishments and Future Plans ", AIAA Space 2011 Conference and Exposition, 2729 Sept. 2011, AIAA 2011-7114.

Leshin, L. A. et al. (2013). "Volatile, Isotope, and Organic Analysis of Martian Fines with the Mars Curiosity Rover." Science (27 Sept. 2013): 341(6153), 1238937.

Mazanek, D. D., Merrill, R. G., Belbin, S. P., Reeves, D. M., Earle, K. D., Naasz, B. J., and Abell, P. A. (2014). "Asteroid Redirect Robotic Mission: Robotic Boulder Capture Option Overview." AIAA SPACE 2014 Conference and Exposition, San Diego, CA, AIAA 2014-4432.

Metzger, P. (2014), private communication.

Muirhead, B. K., and Brophy, J. R. (2014). "Asteroid Redirect Robotic Mission feasibility study." Aerospace Conference, 2014 IEEE, 1-14.

National Aeronautics and Space Administration Authorization Act of 1958 (Public Law 85568), July 29, 1958, title I, Section 102.

National Aeronautics and Space Administration Authorization Act of 2005 (Public Law 109155), January 4, 2005, Section 321, George E. Brown, Jr. Near-Earth Object Survey Act.

NRC (National Research Council) (2010). "Defending Planet Earth: Near-Earth Object Surveys and Hazard Mitigation Strategies." The National Academies Press, Washington, D.C..

Sanders, G. B., and Larson. W. E. (2012). "Progress Made in Lunar In Situ Resource Utilization under NASA's Exploration Technology and Development Program." J. Aerospace Engineering 26(1), 5-17.

Tsuda, Y. (2013). "System design of the Hayabusa 2 - Asteroid sample return mission to 1999JU3.” Acta Astronautica 91, 356-362. 